\title{
PROPUESTA DE PLANEACIÓN ESTRATÉGICA COMO HERRAMIENTA DE COMPETITIVIDAD APLICADA AL EMPRENDIMIENTO COTTON CANDY DE LA CIUDAD DE CARTAGENA DE INDIAS, COLOMBIA
}

\section{STRATEGIC PLANNING PROPOSAL AS A COMPETITIVENESS TOOL APPLIED TO THE COTTON CANDYVENTURE IN THE CITY OF CARTAGENA DE INDIAS, COLOMBIA}

\section{Autor 1}

Amalio Segundo Otero Tapia

DocenteInvestigador - Universidad Nacional Abierta y a Distancia-UNAD

ORCID: https://orcid.org/0000-0002-0142-0344

Correo electrónico: amalio.otero@unad.edu.co

\section{Autor 2}

Engler de Jesús Ariza García

Docente Investigador - Universidad Nacional Abierta y a Distancia-UNAD

ORCID: https://orcid.org/0000-0002-6444-7982

Correo electrónico: engler.ariza@unad.edu.co

\section{Autor 3}

Roy AntonioCastilloGiraldo

Docente Universidad Nacional Abierta y a Distancia -UNAD

ORCID: https://orcid.org/0000-0003-3678-0371

Correo electrónico: roy.castillo@unad.edu.co

\section{Autor 4}

\section{Carlos Mauro Lombana Moron}

Estudiante Universidad Nacional Abierta y aDistancia UNAD

ORCID: https://orcid.org/0000-0002-0697-4098

Correo: cmlombanam@unadvirtual.edu.co

\section{Autor 5}

\section{Jhosman Fuentes Padilla}

Estudiante Universidad Nacional Abierta y aDistancia UNAD

ORCID:https://orcid.org/0000-0002-4357-1458

Correo: jfuentespa@unadvirtual.edu.co 


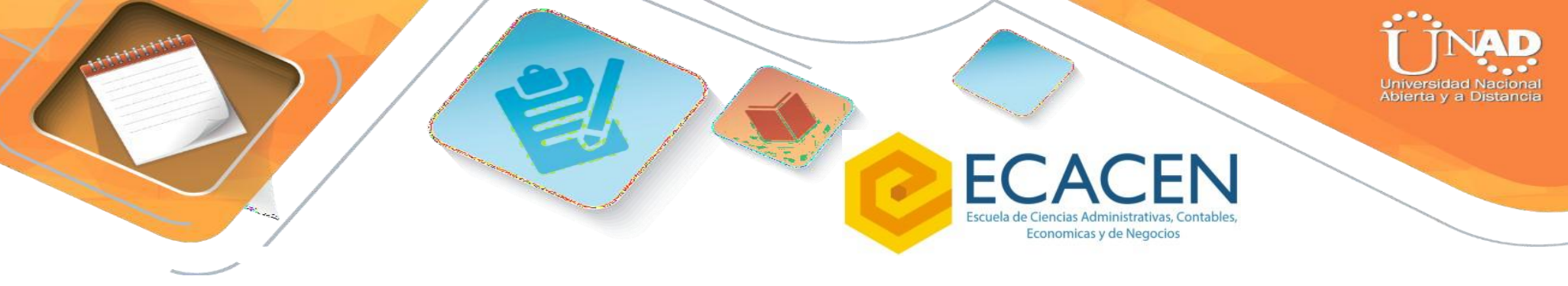

Keywords: Mission, Vision, Strategy, Objectives, Competitiveness, Entrepreneurship

\section{INTRODUCCIÓN}

Según la Organización para la Cooperación y el Desarrollo Económico OCDE (2004) las micro, pequeñas y medianas empresas conforman el $90 \%$ de las empresas y generan más de la mitad del empleo que ofrece la actividad economía empresarial. En Colombia según la Revista Empresarial (2017), de las empresas que emprenden el primer año solo subsisten un $55 \%$ de ellas, el segundo el $41 \%$ y el tercero el $31 \%$, lo que afecta el desarrollo del tejido empresarial colombiano y el bienestar de las familias.

Razón por la cual la planeación estratégica es una herramienta fundamental para lograr que los emprendimientos puedan direccionar sus esfuerzos, crecer y mantenerse en el tiempo.Según Druker (2002)citado por Madrigal Moreno, Madrigal Moreno, \& Guerrero Davalos (2015)"la planeación estratégica es el proceso continuo, basado en el conocimiento más amplio posible del futuro, considerado, que se emplea para tomar decisiones en el presente, lo cual implica riesgos futuros en razón de los resultados esperados", teniendo en cuenta los mencionados autores esto conlleva un trabajo continuo direccionado de acuerdo al horizonte futuro de un ente para la toma de decisiones en el presente. Razón por la cual es una herramienta que pueden utilizar las empresas nacientes que tienen ciertos problemas de organización, especialmente las microempresas, dado que, muchas veces los propietarios no están preparados con el nivel de conocimiento lo que se traduce en problemas para la empresa, ya que al no ser capaces de visionar o de planear su futuro, crecen de manera desordenada sin dirección, lo que al paso del tiempo empiezan a enfrentar situaciones adversas debido a la falta de planeación, por lo que la profundización y planeación en estos temas debe ser muy importante para cualquier empresa que quiera permanecer en el mercado, se toma el caso del emprendimientoCotton Candy, una empresa naciente dedicada a producir y comercializar algodones de azúcar, a la cual se le aplica el 


\section{ECACEN}

\begin{tabular}{|l|l|}
\hline \multicolumn{1}{|c|}{ Fortalezas } & \multicolumn{1}{c|}{ Amenazas } \\
\hline -la empresa se interesa por los & -Escases de algunos materiales \\
procesos de innovación. & por falta de proveedores. \\
-Apoyo de profesionales idóneos. & -Afectaciones debido a la \\
-Variedad de catalogo. & pandemia, lo que no ha permitido \\
-Capacidad de producción. & el crecimiento y promoción de la \\
-Confiabilidad & empresa. \\
-Se estan implementando & -Competencia de otras empresas \\
procesos de mejora continua & en la ciudad. \\
& \\
\hline
\end{tabular}

Fuente: Elaboración propia

Se pudo evidenciar que el emprendimiento posee aspectos negativos por mejorar en su entorno y que a largo plazo pueden afectar seriamente su funcionamiento y crecimiento, pero tambien tiene grandes aspectos positivos que si se potencian pueden contribuir mucho en su direccionamiento y ayudando a corregir los aspectos indeseables, en todo caso se evidencia la importancia de realizar el análisis DOFA dentro del emprendimiento, "esto lepermitirá alempresariola prevención de los efectos de estasvulnerabilidades y, a su vez,darlas a conocer al equipo de trabajo,a fin de que se logre una agrupación de estos elementos, trabajar en su mejora ycontribuir así alfortalecimiento dela estructuraorganizacional."(Ibarguen Mosquera, Rueda Sanchez, \& Garcia Caicedo, 2019).

\section{El cuadro de mando integral:}

Se le puede a tribuir la autoría del concepto de cuadro de mando integral a Rober Kaplan y a David Norton los cuales lo propusieron para un trabajo en una empresa de semiconductores, la idea era presentar una forma diferente de gestionar una empresa de una manera más amplia y no solamente basada en indicadores financieros, Martin lo define como "Es una herramienta que sirve como espejo para que el gerente de la empresa 


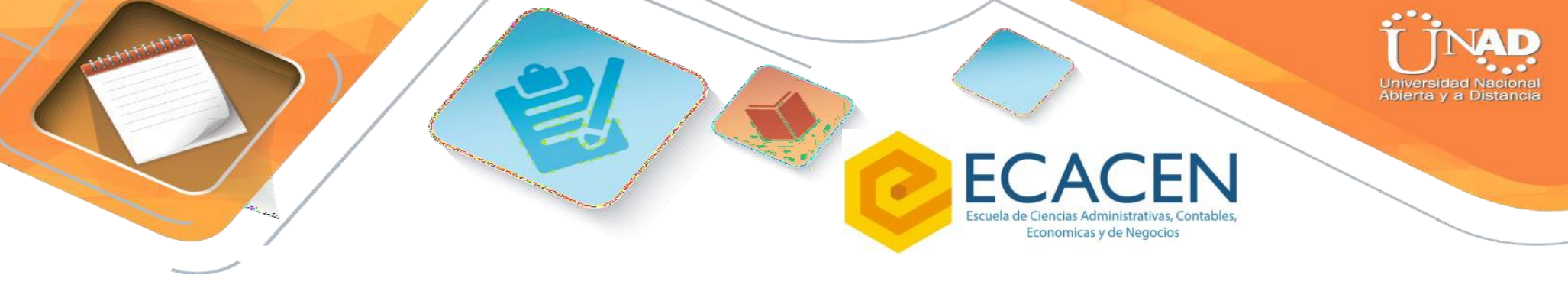

pueda medir si se está cumpliendo lo establecido en la visión y misión de la compañía." (Martin, 2019). Kaplan y Nortonen el año 2000 amplían la metodología del cuadro de mando integral propone utilizar mapas estratégicos con el fin de comprender la estrategia de la empresa de una manera mucho más clara "para poder implementar la estrategia primero debía comprenderse. Por ello se ponen mapas estratégicos que han sido utilizados como mapas mentales o ideas funcionando para la comprensión de la estrategia" (Lasanta, 2020)

Hoy en día aún existen muchas empresas que solo dan i importancia al tema financiero y es el único indicador que utilizan para medir el desempeños de sus organizaciones, la importancia de aplicar la estrategia basada en el cuadro de mando integral es la visión amplia que se le da a la empresa y como articula otros escenarios que son fundamentales para su desarrollo, basándose e integrándose en la visión y en la misión, contribuyendo a que pueda ser alcanzada de una manera más fácil y organizada, en este sentido Kaplan y Norton afirmaron "los indicadores financieros informan sobre los resultados (indicadores tardíos) pero no comunican cuáles serían la causas de los resultados futuros, la causas que indican cómo crear valor a través de las inversiones en clientes, proveedores, empleados, tecnología e innovación" (Panneso Mayor \& Jaramillo Abadia, 2016).

La representación de la estrategia de la empresa por medio del cuadro de mando integral permite no solo dar a conocer de manera clara los objetivos estratégicos sino que también muestra la relación que hay entre ellos y como esta relación puede crear sinergias que facilitan la forma de desarrollarlos, de esta manera se pueden involucrar en el desarrollo de las actividades para alcanzar los objetivos, es una representación gráfica de la estrategia de la empresa representándola de manera coherente, "El mapa estratégico representa la relación de causa-efecto que hay entre los objetivos trazados y los temas estratégicos. Cuando estas relaciones están claramente definidas, se puede comunicar y delegar las responsabilidades en forma efectiva." (Conexionesan, 2017). 


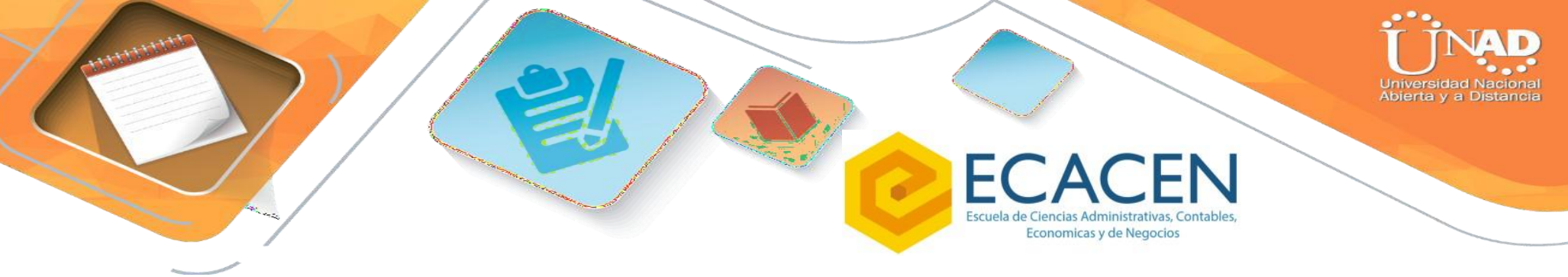

El CMI o BSC ayuda a representar la estrategia de la empresa mediante 4 perspectivas donde se presentas los objetivos a alcanzar por medio de cada una de ellas:

-Perspectiva Financiera: Esta perspectiva describe la forma como la empresa va a alcanzar los objetivos en temas de crecimiento económico.

-Perspectiva Clientes: En esta perspectiva se fijan los objetivos que permiten generar valor al cliente, permitiendo lograr satisfacción, fidelidad y aumento de estos.

-Perspectiva Procesos Internos: Los objetivos fijados en esta perspectiva buscan mejorar los procesos de la empresa con el fin de generar valor en el cliente.

-Perspectiva Aprendizaje: Los objetivos de esta perspectiva busca generar la capacidad para adaptar los procesos internos de la empresa.

A demás de las cuatro perspectivas, también se encuentran las líneas estratégicas las cuales son agrupaciones verticales de objetivos comunes las cuales integran objetivos de cada una de las perspectivas. El cuadro de mando integral se construye de arriba comenzando por la perspectiva financiera, siguiendo por la perspectiva cliente, procesos internos y finalizando por la perspectiva aprendizaje y se ejecuta abajo hacia arriba.

De esta manera teniendo en cuenta el diagnóstico realizado al emprendimiento Cotton Candy se procede a realizar el siguiente cuadro de mando integral: 


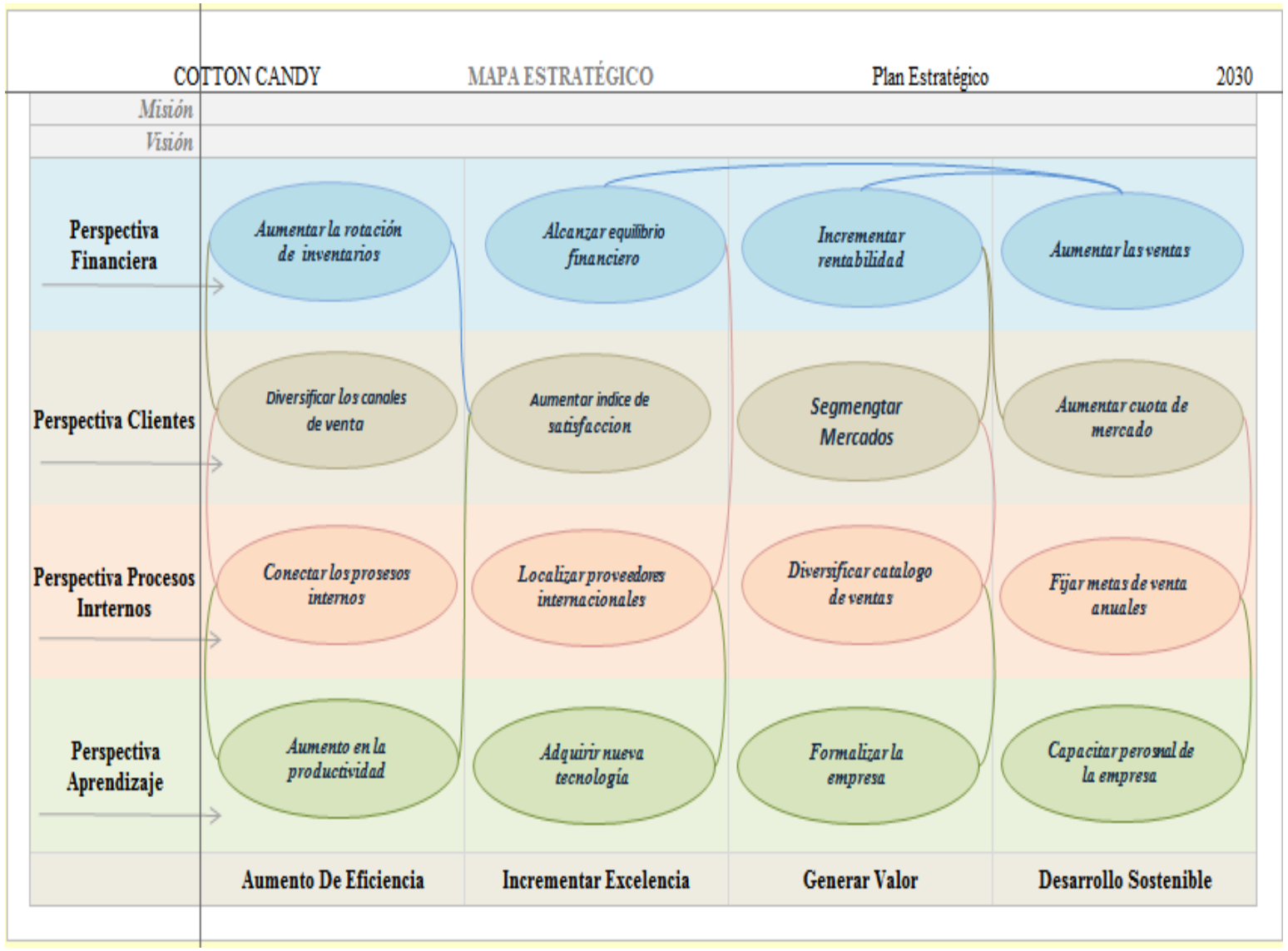

Fuente: elaboración propia basados en Kaplan, Roberto y Norton, David. "Cuadro de Mando Integral". Gestión 2000. Barcelona. 2000.

Estableciendo los objetivos se le puede abrir un camino al emprendimiento para que esta pueda empezar a trabajar de manera aunada y organizada, evitando el desperdicio valioso de recursos, ya que este es uno de los factores por los cuales muchas micro y pequeñas empresas nacientes empiezan a perder competitividad frente a su competencia y desaparecen del mercado, de esta manera se pueden formular metas y actividades claras, asignar recursos valiosos y entrar a competir y posicionarse en el mercado.

\section{RESULTADOS Y DISCUSIÓN}




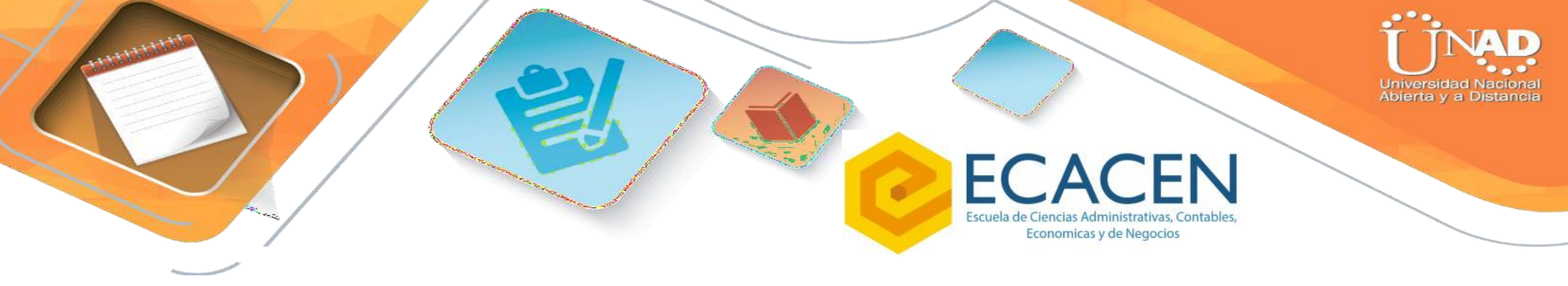

Al analizar el emprendimiento se pudo evidenciar que no contaba con una misión ni visión establecidas, tenía algunas falencias internas que debieron ser corregidas con el fin de fortalecerla y potenciarla, de igual manera el análisis externo de esta mostraba que estaba en desventaja con la competencia, pero tenía muchas posibilidades y oportunidades debido al amplio mercado y que existían pocos competidores. Una vez realizadas las herramientas de diagnostico se procedió a la construcción de objetivos estratégicos basados en el cuadro de mando integral, lo que dejo un camino muy evidente para el crecimiento de la empresa.

Es importante para fortalecer el trabajo aplicar otras herramientas como la matriz del perfil competitivo para saber cual es la posición de la empresa frente a otros competidores y el cuadro de posicionamiento estratégico para medir como esta la empresa en el sector, sin embargo el realizar el diagnostico y crear el cuadro de mando integral es un gran avance que le permitirá a la empresa direccionar sus esfuerzo y crear una ruta que le permita mejorar y empezar su camino al crecimiento y desarrollo organizacional.

Lo que se puede asegurar que cualquier empresa que no aplique planeación estratégica a su empresa y que no se preocupe por establecer una visión por la cual perseguir, con el tiempo empezara a perder competitividad y corre el riesgo de desaparecer del mercado, ya que, estaría en desventaja frente a la competencia que si lo hace y que día a día se preocupa por mejorar y posicionarse en el mercado.

\section{CONCLUSIÓN}

Una vez aplicadas las herramientas de la planeación estratégica, se logró construir una propuesta del plan estratégico para el emprendimiento Cotton Candy mediante la información suministrada por el emprendedor y el apoyo de la bibliografía disponible sobre el tema. Los resultados indican que cuando las empresas no son direccionadas se produce un desperdicio de recursos y se puede estar en desventaja ante la 
competencia, sin embargo, construir un mapa estratégico le permite a un emprendimiento aprovechar mejor los recursos y direccionarlos a la consecución de objetivos, logrando el crecimiento de la empresa y se puede lograr un posicionamiento en el mercado.

\section{REFERENCIAS}

Conexionesan. (2017). Apuntes Empresariales. ¿Qué es un mapa estratégico y qué utilidad tiene en la organización? https://www.esan.edu.pe/apuntes-empresariales/2017/07/que-esun-mapa-estrategico-y-que-utilidad-tiene-en-la-organizacion/

Ibarguen Mosquera, Y. Y., Rueda Sanchez, L. K., \& Garcia Caicedo, A. M. (2019). Análisis de la estructura organizacionaldelconsultorio Innovadentala partir de la matriz DOFA. PLOUTOS , 19.

Kaiten, J. C. (2017). Importancia de la Visión y Misión dentro de las organizaciones. Importancia de la Visión y Misión dentro de las organizaciones.

http://biblioteca.udgvirtual.udg.mx/jspui/handle/123456789/1087

Kaplan, R. \& Norton, D. (2000). "Cuadro de Mando Integral". Gestión 2000. Barcelona.

Lasanta, I. (2020). Iedge Bussines School. Historia y evolución del cuadro de mando integral (CMI) o "Balanced Scorecard". https://www.iedge.eu/ines-lasanta-historia-evolucion-del-cuadrode-mando-integral-cmi-balanced-scorecard

Madrigal Moreno, F., Madrigal Moreno, S., \& Guerrero Davalos, C. (2015). Planeacion estrategica y gestion de conocimiento en las pequeñas y medianas empresas(PYMES), herramientas basicas para su permanencia y consolidacion. European Scientific Journal , 12. 
Martin, J. M. (2019). ¿Qué es y para qué sirve un cuadro de mando integral o 'balanced scorecard'? España: UNIR.

Martínez Salgado (2012). El muestreo en investigación cualitativa.

Principios básicos y algunas controversias. Ciencia \&Saúde Colectiva, 17, 613-619.

Organización para la Cooperación y el Desarrollo Económico (OCDE) (2004). Promotingentrepreneurship and innovativeSMEs in a global economy. Towards a more responsible and inclusive globalization, Second OCDE Ministerial ConferenceonSMEs, 2-5 junio, Estambul.

Panneso Mayor, C. O., \& Jaramillo Abadia, A. B. (2016). Universidad Libre. Modelo balanced scorecard para el instituto de educación. https://repository.unilibre.edu.co/bitstream/handle/10901/9613/] aramillo_Panesso_2016.pdf?sequence $=1$

Revista Empresarial \& Laboral. (2017). Cifras de Emprendimiento en las empresas Colombianas. https://revistaempresarial.com/empresas/pymesempresas/emprendimiento-pymes/cifras-emprendimientoempresas-colombianas/ 